\title{
Warm-hot intergalactic baryons revealed
}

\author{
L. Zappacosta ${ }^{1}$, F. Mannucci ${ }^{2}$, R. Maiolino ${ }^{3}$, R. Gilli ${ }^{3}$, A. Ferrara ${ }^{3}$, A. Finoguenov ${ }^{4}$, N. M. Nagar ${ }^{3}$, and D. J. Axon ${ }^{5}$ \\ 1 Dipartimento di Astronomia e Scienza dello Spazio, Largo E. Fermi 2, 50125 Firenze, Italy \\ 2 Centro per l'Astronomia Infrarossa e lo studio del mezzo interstellare (CAISMI), CNR, Largo E. Fermi 5, \\ 50125 Firenze, Italy \\ 3 Osservatorio Astrofisico di Arcetri, Largo E. Fermi 5, 50125 Firenze, Italy \\ 4 Max-Planck-Institut für extraterrestrische Physik, Giessenbachstraße, 85748 Garching, Germany \\ 5 Department of Physical Sciences, University of Hertfordshire, College Lane, Hatfield, Hertfordshire AL10 9AB, UK
}

Received 6 June 2002 / Accepted 29 July 2002

\begin{abstract}
Several popular cosmological models predict that most of the baryonic mass in the local universe is located in filamentary and sheet-like structures associated with galaxy overdensities. This gas is expected to be gravitationally heated to $\sim 10^{6} \mathrm{~K}$ and therefore emitting in the soft X-rays. We have detected diffuse soft X-ray structures in a high Galactic latitude ROSAT field after point source subtraction and correction for Galactic absorption. These diffuse structures have an X-ray energy distribution that is much softer than expected from clusters, groups or unresolved emission from AGNs, but are consistent with that expected from a diffuse warm intergalactic medium. To discriminate between a Galactic or extragalactic nature of the diffuse gas we have correlated the soft X-map with multiband optical images in this field. We have found a significant overdensity of galaxies in correspondence with the strongest diffuse X-ray structure. The photometric redshift distribution of the galaxies over the $\mathrm{X}$-ray peak has an excess over field galaxies at $z \sim 0.45$. This result strongly suggests that the diffuse $\mathrm{X}$-ray flux is due to extragalactic emission by warm gas associated with an overdense galaxy region at $z \sim 0.45$.
\end{abstract}

Key words. large-scale structure of Universe - X-rays: diffuse background

\section{Introduction}

The mismatch between the density of baryons observed in the local Universe and the baryon density observed and predicted at high redshift is currently one of the most puzzling issues in cosmology. While the density of baryons, $\Omega_{\mathrm{b}}$, observed in stars and gas $(\mathrm{HI}+\mathrm{HII})$ in the local Universe does not exceed 0.01 (Fukugita et al. 1998), observations of the Ly $\alpha$ forest at $z=2$ (Rauch et al. 1997) and Big Bang nucleosynthesis constraints (Burles \& Tytler 1998) both give $\Omega_{\mathrm{b}} \simeq 0.04$ or larger (Pettini \& Bowen 2001). One possibility is that at $z<1$ the baryonic gas falls onto the cosmic web pattern and is heated by shock mechanisms forming filamentary and sheet-like structures (Cen \& Ostriker 1999; Davé et al. 2001). Such diffuse gas, called Warm-Hot Intergalactic Medium (WHIM), should be detectable in the soft X-rays as a consequence of having a temperature in the range $10^{5} \mathrm{~K}<T<10^{7} \mathrm{~K}$. A tight connection with the filamentary distribution of galaxies is expected from $N$-body simulations (see Bond et al. 1996 for a theoretical discussion).

Various models of diffuse X-ray emission have been proposed by several authors during the past year (Phillips et al. 2001; Kuntz et al. 2001; Bryan \& Voit 2001); nonetheless, observational evidence for diffuse/filamentary X-ray emission is

Send offprint requests to: $\mathrm{R}$. Maiolino,

e-mail: maiolino@arcetri.astro.it still sparse. Wang \& McCray (1993) found an excess of emission in some ROSAT fields which they ascribed to a diffuse component of the X-ray background due to WHIM. Soltan et al. (1996) detected a correlation signature between the soft $\mathrm{X}$-ray background and galaxies. Filamentary soft X-ray structures were identified by Warwick et al. (1998) in various overlapping ROSAT pointings near the Lockman Hole. Structures laid among clusters were found by Kull \& Böhringer (1999) in the core of the Shapley Supercluster and Tittley \& Henriksen (2001) along the line of sight connecting two Abell clusters. Scharf et al. (2000) presented tentative evidence of a soft X-ray filamentary structure which seems to correlate with the density of galaxies measured in the I-band. Recently, Bagchi et al. (2002) pointed out the discovery of a presumably filamentary structure both in radio and soft X-ray traced by a galaxy arc.

We have re-analyzed several ROSAT pointings toward a region close to the Lockman Hole with exposure times $>10 \mathrm{ksec}$ and, after removal of the point sources and correction for Galactic absorption, we have detected diffuse X-ray emission. We have started a program of optical multiband wide field imaging of the regions showing diffuse X-ray emission with the goal of detecting associated galaxy overdensities, which would support the extragalactic nature of the large scale soft X-ray structures. 


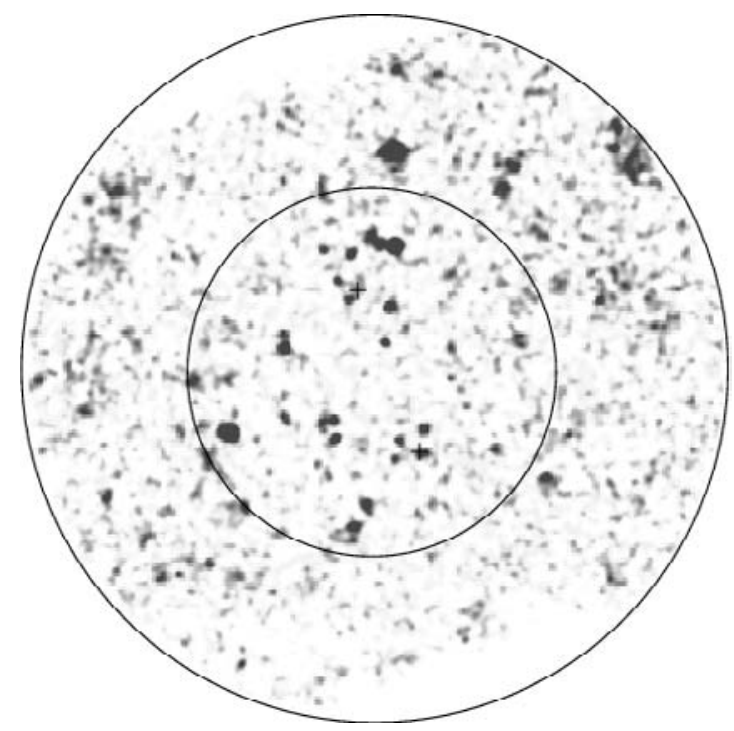

(a)

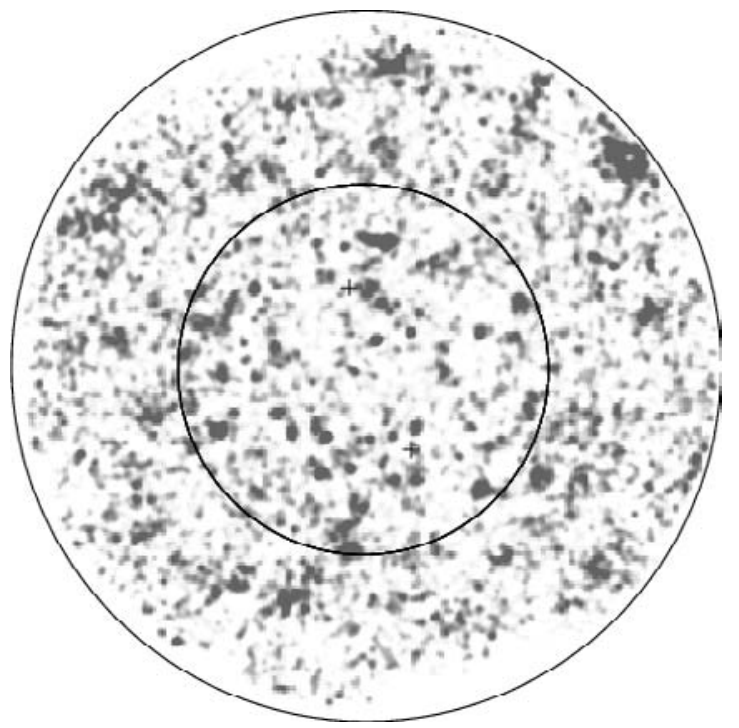

(b)

Fig. 1. Panel a) Soft (R2, 0.14-0.284 keV) map smoothed to 15 arcsec showing the point sources; b) Hard (R6+7, 0.73-2.04 keV) map with the same smoothing. The inner circle (1 deg in diameter) encloses the region where the point source removal is reliable. The two crosses indicate the location of the structures discussed in Fig. 2.

In this paper we report preliminary results on one of the ROSAT fields for which we have obtained HI radio data and which has been partially mapped in five optical bands. We present the discovery of a galaxy overdensity at a photometric redshift $z \sim 0.45$ spatially coincident with the most prominent diffuse X-ray structure in the ROSAT field.

The plan of the paper is as follows: in Sect. 2 we discuss the analysis of the X-ray ROSAT maps, in Sect. 3 the reduction of the radio data and the $\mathrm{HI}$ absorption correction for X-ray maps are presented, in Sect. 4 we discuss the optical images analysis. Section 5 contains the photometric redshift estimate and, finally, in Sect. 6 a summary of the results is presented.

\section{X-ray maps}

We have retrieved from the ROSAT archive the images of fields contiguous to the Lockman Hole used by Warwick et al. (1998) to create a large scale soft X-ray map. These authors noted, after subtraction of the point sources, large scale diffuse structures in the $\sim 0.1-0.3 \mathrm{keV}$ band. We have focused on one of the ROSAT fields used by Warwick et al. and, more specifically, on "Field 4", centered at RA(J2000) $10^{\mathrm{h}} 10^{\mathrm{m}} 14^{\mathrm{s}}$ and Dec(J2000) $+51^{\circ} 45^{\prime} 00^{\prime \prime}$, obtained with a PSPC integration of $20 \mathrm{ksec}$. We extracted the images in the various ROSAT bands ${ }^{1}$ by means of the software described in Snowden et al. (1994), which is optimized for the analysis of extended structures in the ROSAT maps. In this way accurate maps of the background and of the effective exposure over the whole field of view can be obtained. We mostly focused on the soft image obtained in the R2 band (PI channels 21 to 40), which samples the energy range $0.14-0.28 \mathrm{keV}$. However, we also use the harder bands (R4+5, 0.44-1.21 keV; R6+7, 0.73-2.04 keV), both for the

\footnotetext{
${ }^{1}$ In the following we will refer to such broad bands energy using the conventional definitions reported in Snowden et al. (1994).
}

point sources removal and to constrain the nature of the diffuse emission. On the R2 image obtained with Snowden's code the detection of extended structures has been performed with two different methods:

1. We have constructed a map of the extended structures with an algorithm based on SExtractor (Bertin \& Arnouts 1996). Our aim is to identify and subtract the point like sources which resolve most of the $0.5-2.0 \mathrm{keV}$ background. This algorithm identifies the point sources by increasing the size of the detection filter with the distance from the center to match the broadening of the ROSAT PSF with the off-axis angle. The detected point sources in R2 and R6+7 bands are subtracted from the soft image and replaced with the surrounding background.

Figure 1 shows the images, before the point sources removal and preliminarily smoothed with a Gaussian kernel of 15 arcsec (i.e. one PSPC pixel), in the soft (R2; Fig. 1a) and the hard (R6+7; Fig. 1b) bands. The soft image was then smoothed with a Gaussian kernel of 5.9 arcmin $F W H M^{2}$ and is shown in Fig. 2a. The two main structures around the center of the field are statistically significant to a level of $\sim 8 \sigma$ (assuming Poisson statistics).

2. To assess the significance of the structures found with the previous method we have also used a wavelet algorithm developed by Vikhlinin et al. (1998). The aim of the wavelet transform in this context is the complete background subtraction to emphasize structures of certain angular size. The idea is to convolve an astronomical image with a kernel that is the difference of two Gaussians, the first with sigma $a$, the second subtracted from the previous with sigma $b=2 a$.

\footnotetext{
2 This specific smoothing width was chosen because this is the minimum size of structures found with the second method. A FWHM of 5.9 arcmin corresponds to Gaussian with a $\sigma$ of 10 pixels.
} 


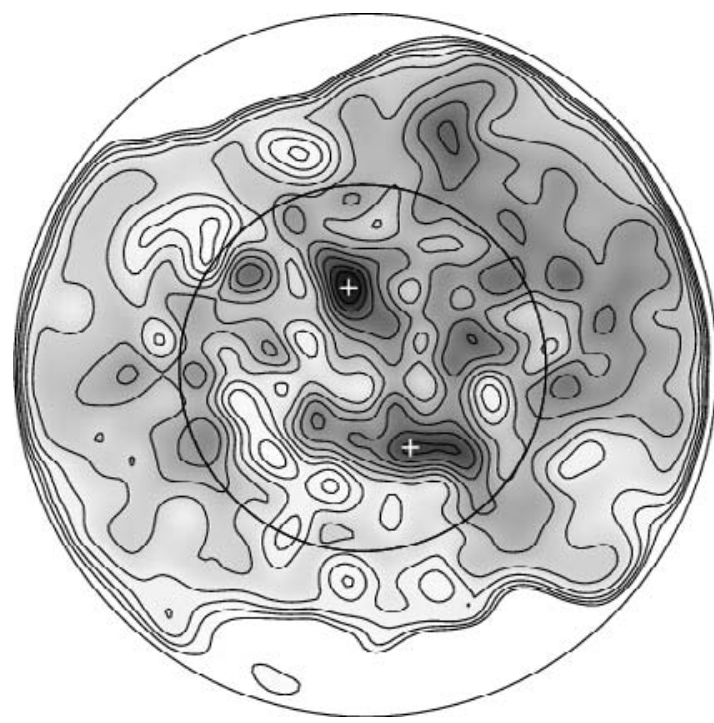

(a)

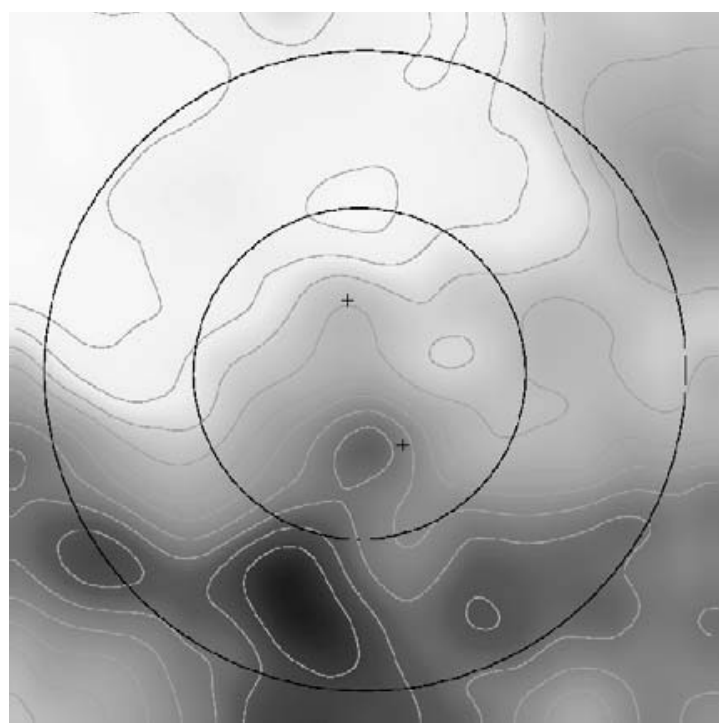

(c)

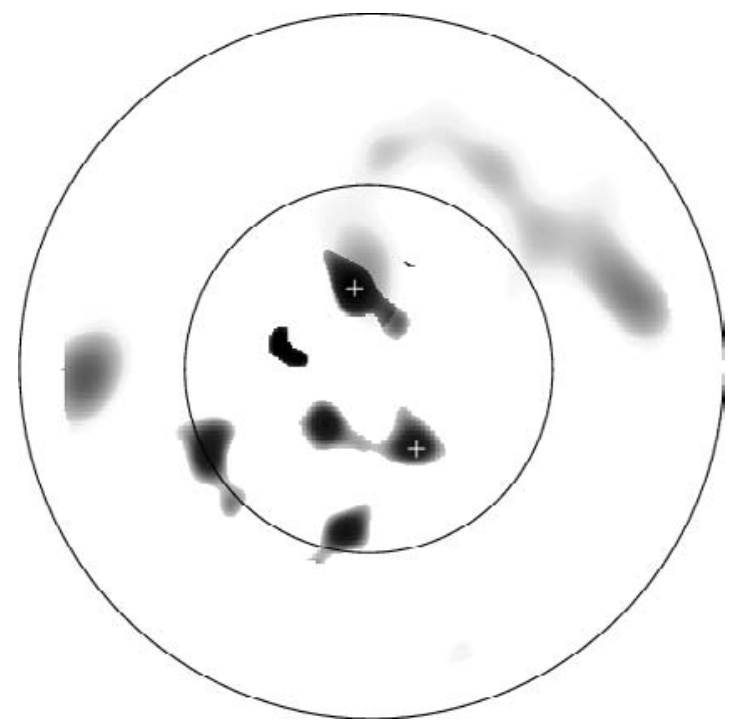

(b)

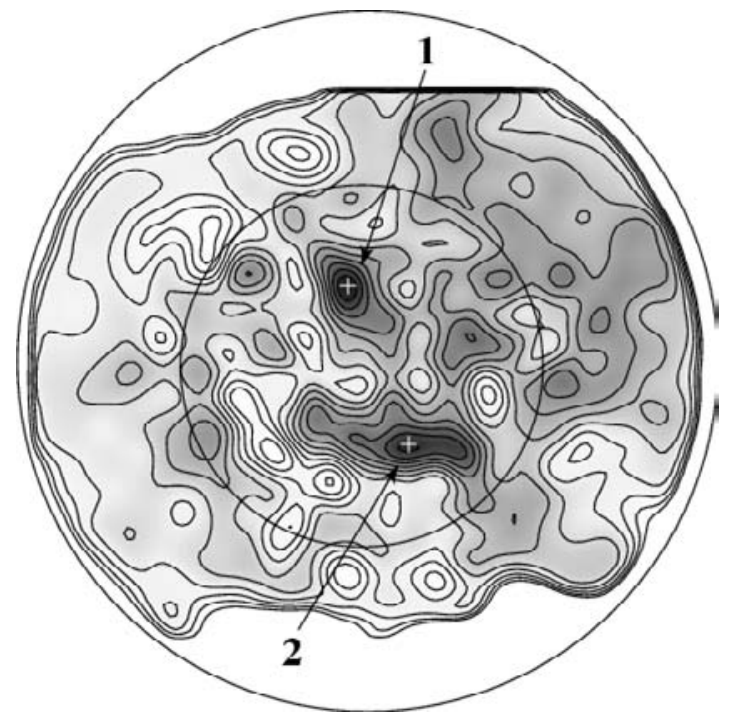

(d)

Fig. 2. a) Soft $X$-ray map smoothed to a resolution of 5.9 arcmin after removing the point sources identified in the R 2 and R6+7 maps; b) Wavelet map showing extended structures with high $(4 \sigma)$ statistical significance; c) Map of Galactic HI column density (contours from 5.3 to $10.7 \times 10^{19} \mathrm{~cm}^{-2}$, spaced by $0.6 \times 10^{19}$ ), lighter gray areas correspond to lower HI columns; d) Soft map corrected for HI absorption. As in Fig. 1 the inner circle ( $1 \mathrm{deg}$ in diameter) encloses the region where the point source removal is reliable. The two structures, marked with crosses, are in a region where the ROSAT PSF is only $30 \%$ worse than that in the center.

In the following the kernel sizes will be reported as $a$. We require the extended emission to be detected at a level of $4 \sigma$, with respect to the background fluctuations, and each detection followed down to the $1 \sigma$ level. Given the strong radial variation of the ROSAT PSF, not implemented into the wavelet algorithm, the structures detected with wavelet kernel size less than 0.75 were considered as point sources, while those detected by wavelet filters larger than 1.00 are considered as extended. Features detected by wavelet filter sizes between 0.75 and 1.00 are to be considered point sources in the outer parts of the ROSAT field (and therefore subtracted), whereas they were considered to be potentially extended in the inner parts. In Fig. $2 b$ we show the map of structures detected with wavelet kernel sizes in the range $0.75-1.25$ for the soft ROSAT band R2. The advantage of the wavelet detection algorithm is that it provides a quantitative measure of the statistical reliability of detected extended structures with respect to background fluctuations. The size measured for the smallest diffuse structure is $\sim 5.9$ arcmin.

The most prominent diffuse/extended structures are in common to both methods at least in the central part, where the detection 


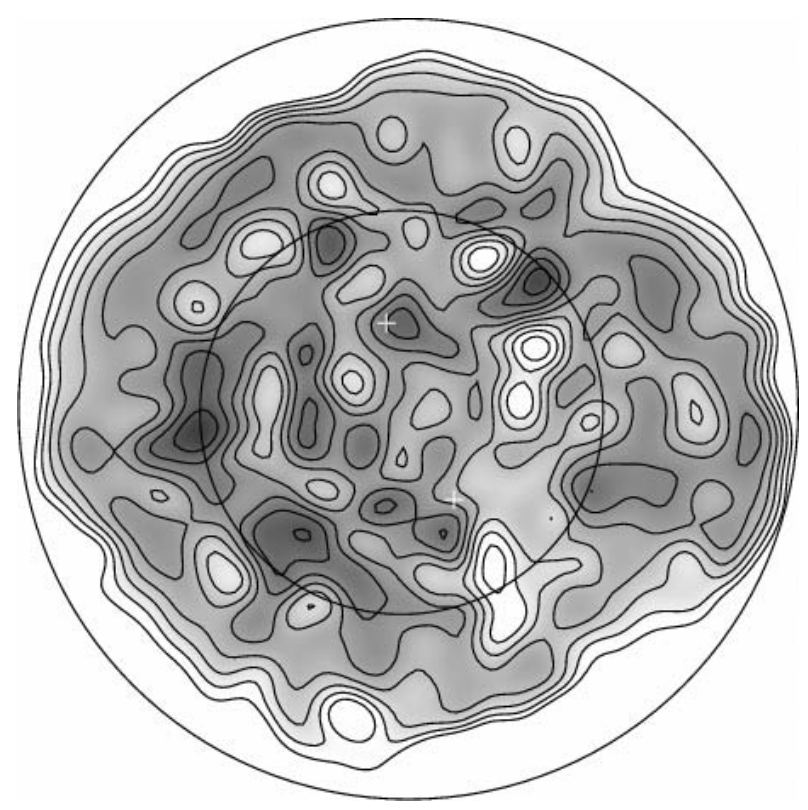

Fig. 3. Map of the extended hard emission (R6+7 band) obtained by removing the point sources and smoothing to a resolution of 5.9 arcmin.

of point sources is more secure, indicating that they are not artifacts of the specific algorithm. We mark as " 1 " and " 2 " in Fig. 2d the two most prominent structures in the central region. In the outer parts, where the ROSAT PSF is much wider, the identification of the extended structures is less reliable; however, even in these outer parts there is reasonable agreement between the maps obtained with the two methods. Finally, as already commented on by Warwick et al. (1998), we note that the same diffuse structures are found by using other offset ROSAT pointings which partly overlap this field, lending further weight to the argument that they are not instrumental artifacts.

In Fig. 3 we show the map of the diffuse emission detected in the hard band $(\mathrm{R} 6+7)$ obtained by removing the point sources detected with SExtractor and by smoothing to a resolution of 5.9 arcmin (this is the hard-band analogous of Fig. 2a). With the exception of structure " 1 ", which shows some faint hard emission as discussed below, the diffuse/extended emission observed in the hard band $(\mathrm{R} 6+7)$ is not correlated with the diffuse emission structures observed in the soft R2 band. This indicates that the diffuse features observed in the soft map are related to structures with a much softer emission than that due to clusters or AGNs and, in particular, cannot be ascribed to unresolved discrete sources which make the $0.5-2 \mathrm{keV}$ background.

A comparison between soft and medium $(\mathrm{R} 4+5)$ maps shows a weak correlation as expected from the hard tail of the diffuse WHIM emission.

In Fig. 4 we plot the fluxes ${ }^{3}$ in three bands (R2, R4+5, $\mathrm{R} 6+7$ ) of the brightest diffuse structure (marked as " 1 " in Fig. 2d), and the expected spectra of various classes of sources: type 1 AGNs making the $0.5-2 \mathrm{keV}$ background, groups of

\footnotetext{
3 The uniform contribution from the Local Bubble (Snowden et al. 1998) was subtracted from all images and the soft X-ray emission was corrected for Galactic absorption as discussed in Sect. 3.
}

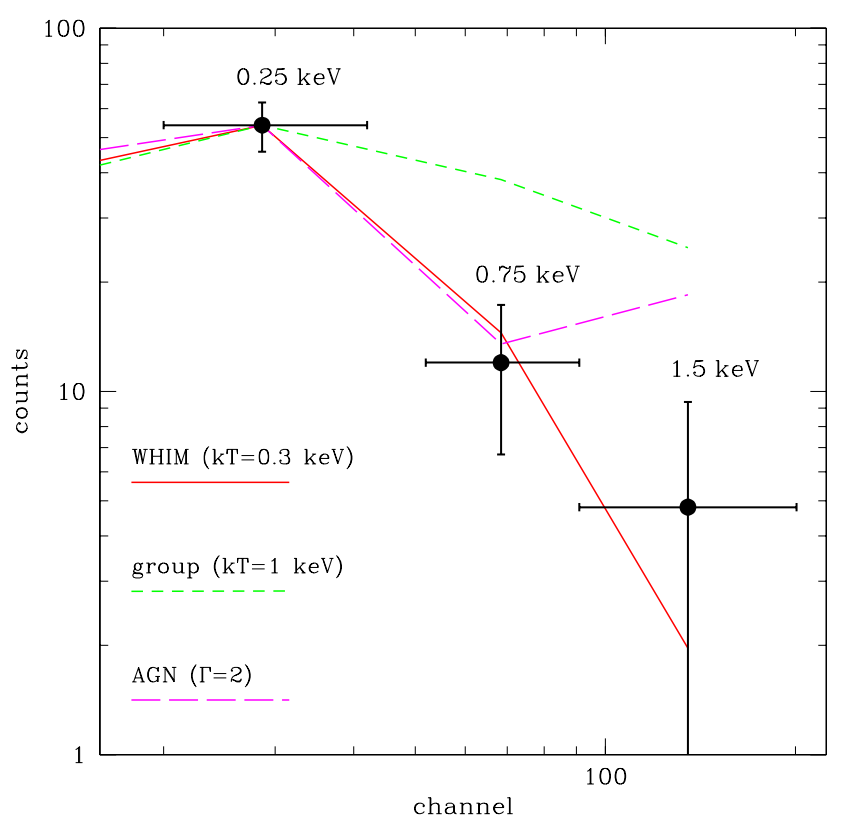

Fig. 4. The spectral shape of the strongest filament (marked " 1 " in Fig. 1d) compared with that expected from WHIM and groups of galaxies at $z=0.45$ and type 1 AGNs (see Sect. 2). The points are the measurements obtained by maps in R2, R4+5 and R6+7 band by extracting the total counts within an aperture of 7 arcmin around the peak. The horizontal errorbars refer to the ROSAT channels, while the bands partly overlap in energy as discussed in Snowden et al. (1994).

galaxies at $T=10^{7} \mathrm{~K}$ (clusters would be even hotter), and thermal emission by the WHIM at $T=3 \times 10^{6} \mathrm{~K}$. For both the groups and the WHIM we adopted a 0.3 solar abundance and $z=0.45$ (see Sect. 5 for the specific choice of this redshift). For AGNs we assumed a power-law with $\Gamma=2$. The choice of $\Gamma$ is lower than the canonical $\Gamma=2.5$ obtained from shallow ROSAT surveys (Walter \& Fink 1993; Brinkmann et al. 2000), but is typical of deeper surveys (Mittaz et al. 1999) like ours. Furthermore it is similar to the mean spectrum of the point sources in our maps ( $\Gamma=2$ for point sources selected in R2 map and $\Gamma=1.7$ for those selected in R6+7 map). Figure 4 shows that the detected emission is inconsistent with emission by clusters or unresolved AGNs, but is fully consistent with thermal emission from the WHIM. It also indicates that the diffuse emission cannot be ascribed to possible residual wings of the PSF of point sources (AGNs) after their subtraction.

The one reported here is one of the first detections of WHIM at intermediate redshift. The diffuse X-ray emission observed in our ROSAT maps can be compared with the (projected) emission expected by the cosmological simulations of a WHIM in the soft X-ray (e.g. Croft et al. 2001). We used simulated images in the $0.2-0.3 \mathrm{keV}$ band, kindly provided to us by R. Croft (private communication), and smoothed them to the angular resolution of our ROSAT maps (Sect. 2). The average emission in the simulations is $\left\langle F_{0.2-0.3 \mathrm{keV}}\right\rangle \approx 7.5 \times$ $10^{-13} \mathrm{erg} \mathrm{cm}^{-2} \mathrm{~s}^{-1} \mathrm{deg}^{-2}$ with $1 \sigma$ fluctuations of about $2 \times$ $10^{-13} \mathrm{erg} \mathrm{cm}^{-2} \mathrm{~s}^{-1} \mathrm{deg}^{-2}$. The average diffuse emission in the central region of our ROSAT map is $10^{-12} \mathrm{erg} \mathrm{cm}^{-2} \mathrm{~s}^{-1} \mathrm{deg}^{-2}$ 
(extrapolated in the $0.2-0.3 \mathrm{keV}$ band), which is in good agreement with the simulations. Taken in conjunction the results of the analysis described above strongly imply that the diffuse structures described here represent one of the first potential detections of a WHIM at intermediate redshift.

At a redshift of $\sim 0.45$ (Sect. 5) the linear extension of the brightest diffuse structure (1) corresponds to a physical size of about $7 \mathrm{Mpc}^{4}$.

\section{Galactic HI maps}

The very soft R2 band (0.14-0.28 keV), where the diffuse structures are more prominent, is strongly affected by even a low amount of absorbing gas along the line of sight. As a consequence, even though our field is close to the Lockman Hole (Lockman et al. 1986) where the Galactic absorption has a minimum $\left(N_{\mathrm{H}} \sim 5 \times 10^{19} \mathrm{~cm}^{-2}\right)$, a major concern is that the observed structures might result from inhomogeneous distribution of Galactic HI clouds along the line of sight. To exclude this possibility we observed "Field 4" at $21 \mathrm{~cm}$ with the Effelsberg 100 meter radio telescope during October 2001.

This telescope has a HPBW (half power beam width) of 9' at $21 \mathrm{~cm}$, which is close to the resolution of our ROSAT maps. We used the $18-21 \mathrm{~cm}$ HEMT 2 channel receiver $\left(T_{\text {sys }} \sim 25 \mathrm{~K}\right)$ and the 1024 channel autocorrelator ${ }^{5}$. The frequency resolution was set to $1.29 \mathrm{~km} \mathrm{~s}^{-1}$ per channel giving a complete velocity coverage of $-380 \mathrm{~km} \mathrm{~s}^{-1}$ to $+280 \mathrm{~km} \mathrm{~s}^{-1}$. This should cover the HI emission from all Galactic and most HVC sources. The target fields were observed in a raster pattern with spacing 4". 3 to Nyquist sample the beam. Frequency switching was used with a $60 \mathrm{~s}$ integration at each raster position (i.e. an effective integration time of $30 \mathrm{~s}$ per raster position). The weather was exceptionally clear for most of the run.

The raw data were initially calibrated using the CLASS and SPEC2 software, converted to $T_{\text {sys }}$, and then corrected for stray radiation using the standard Effelsberg data reduction procedure (Kalberla et al. 1982, 1980). Observations of the Galactic reference field S7 (taken every 2 to $2.5 \mathrm{hrs}$ ) were then used to set the flux scale. Each raster spectrum was first Hanning smoothed to half its resolution and then the "baseline", i.e. the shape of the continuum in each spectrum, was subtracted by using a fourth order polynomial fit. The HI emission between $-100 \mathrm{~km} \mathrm{~s}^{-1}$ and $66 \mathrm{~km} \mathrm{~s}^{-1}$ were summed to produce the total $\mathrm{HI}$ emission in $\mathrm{K} \mathrm{km} \mathrm{s}^{-1}$ at each raster point. This value was multiplied by $1.8 \times 10^{18}$ in order to obtain the total $\mathrm{HI}$ column in $\mathrm{cm}^{-2}$ towards each raster point (i.e. we assumed the HI emission is optically-thin).

In Fig. $2 \mathrm{c}$ we show the resulting $N_{\mathrm{H}}$ map. There is no correlation between the soft $\mathrm{X}$-ray emission and the distribution of $N_{\mathrm{HI}}$ on the scales of $\sim 10-20$ arcmin, indicating that the diffuse structures observed in the former are not due to patchy HI absorption. Some weak anticorrelation is present on the large scales between the smooth North-South gradient of $N_{\mathrm{H}}$ and the very extended X-ray emission (correlation coefficient

\footnotetext{
4 Assuming $H_{0}=70, \Omega=1, \Lambda=0$.

5 The newer 8192 channel autocorrelator was not used due to interference problems at $21 \mathrm{~cm}$.
}

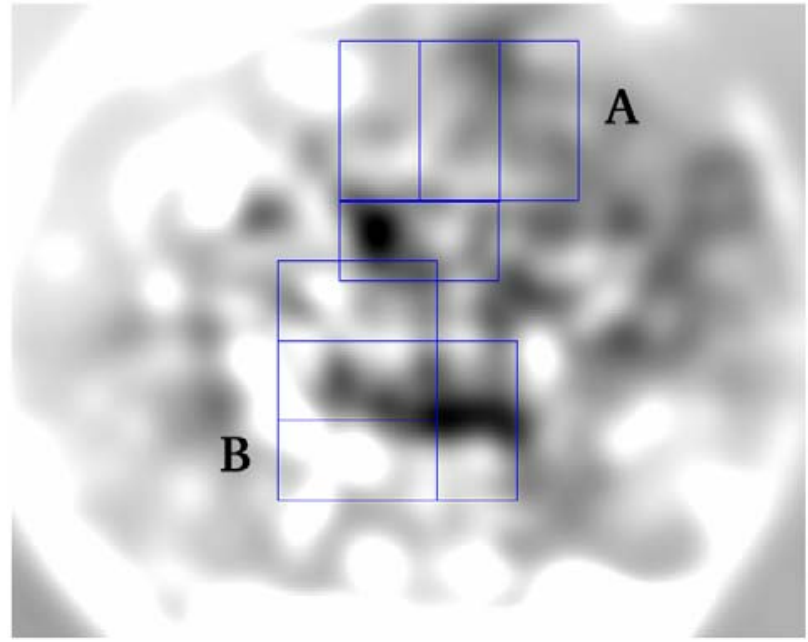

Fig. 5. Position of the WFC pointings over the R2 map.

$r \approx-0.3$ ); this anticorrelation is dominated by the southern $\sim 30^{\prime \prime}$ of the field, i.e. outside the central region which we are investigating, where a significant $\mathrm{HI}$ absorption is present. If this southern part is removed the anticorrelation disappears $(r \approx+0.14)$. The HI map was used to correct the R2 X-ray map for absorption; the absorption-corrected map is shown in Fig. 2d.

\section{The spatial distribution of galaxies in the field}

To map the galaxy distribution we have imaged a subsection of the ROSAT field in the optical. Indeed, if the diffuse emission in the ROSAT maps is due to WHIM, then a spatial correlation with galaxy overdensities is expected.

The optical data were obtained with the Wide Field Camera (WFC) at the Isaac Newton Telescope in service mode on 23 May 2000 and on 15 March 2001. This camera has 4 CCDs covering a field of $\sim 34^{\prime} \times 34^{\prime}$ arcmin (with a gap of $\sim 10^{\prime} \times$ $10^{\prime}$ arcmin due to the distribution of the CCDs), in five broad band filters: $U$ (RGO), $B$ (Kitt Peak), $V$ (Harris), $r^{\prime}$ (Sloan) and $i^{\prime}$ (Sloan). Two fields in the central region of the ROSAT map were observed; the location of the two WFC pointings is shown in Fig. 5: the northern field (field A hereafter) was observed on March-2001, while the southern field (field B hereafter) was observed on May-2000. The seeing was about 1.3" and 1.6" during observations of field A and field B, respectively.

For each field we have performed standard procedures of de-biasing, flat fielding, correction of non linearity and, in the case of $i^{\prime}$, correction for fringes. We used SExtractor to build a preliminary galaxy catalog. The algorithm was run on the $r^{\prime}$ images as they are the most sensitive, at least for galaxies in the redshift range of interest. We have detected 10877 objects in field $\mathrm{A}$ and 8650 in field $\mathrm{B}$.

The zero points were determined by the observation of the standard Landolt fields sa104 and sa101 (Landolt 1992). However, to achieve the maximum accuracy in the photometric calibration we refined the zero points with colorcolor diagrams. This method consists of plotting the brightest non-saturated stars (in our fields those with $17<m\left(r^{\prime}\right)<20$ 


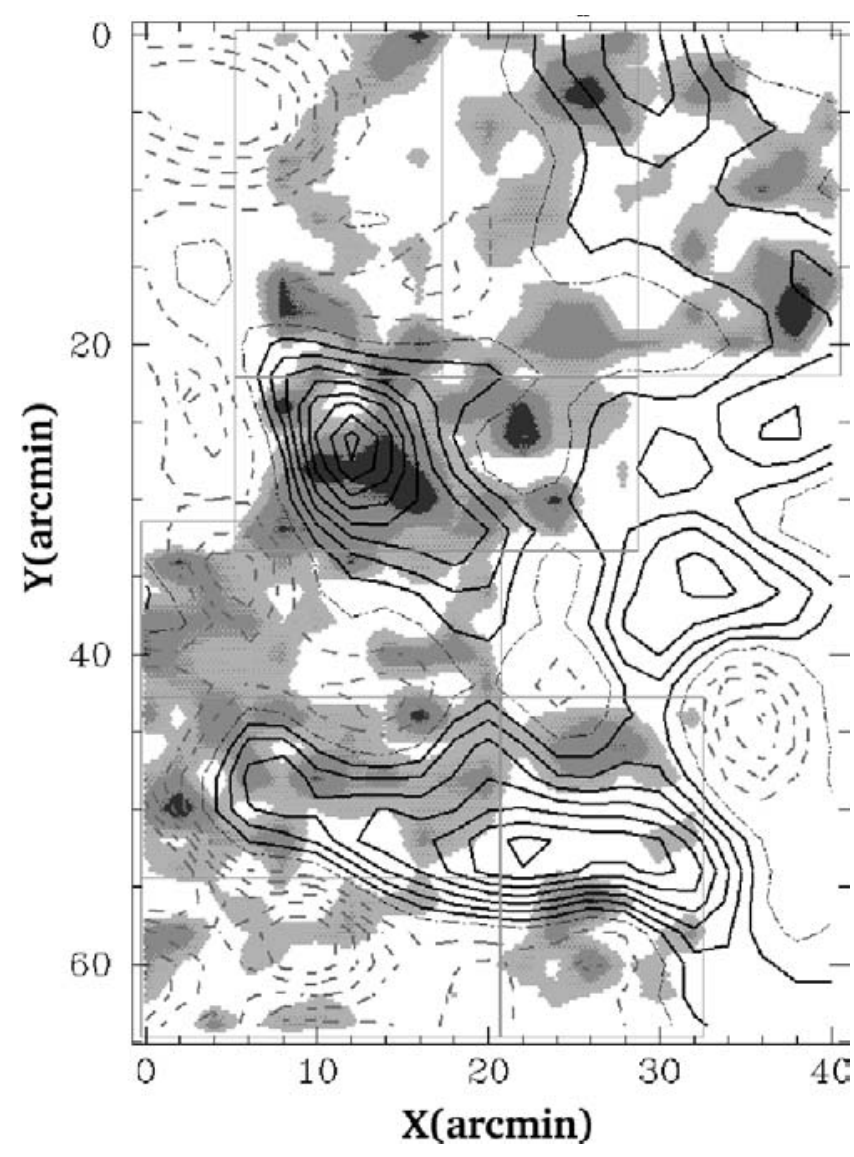

(a)

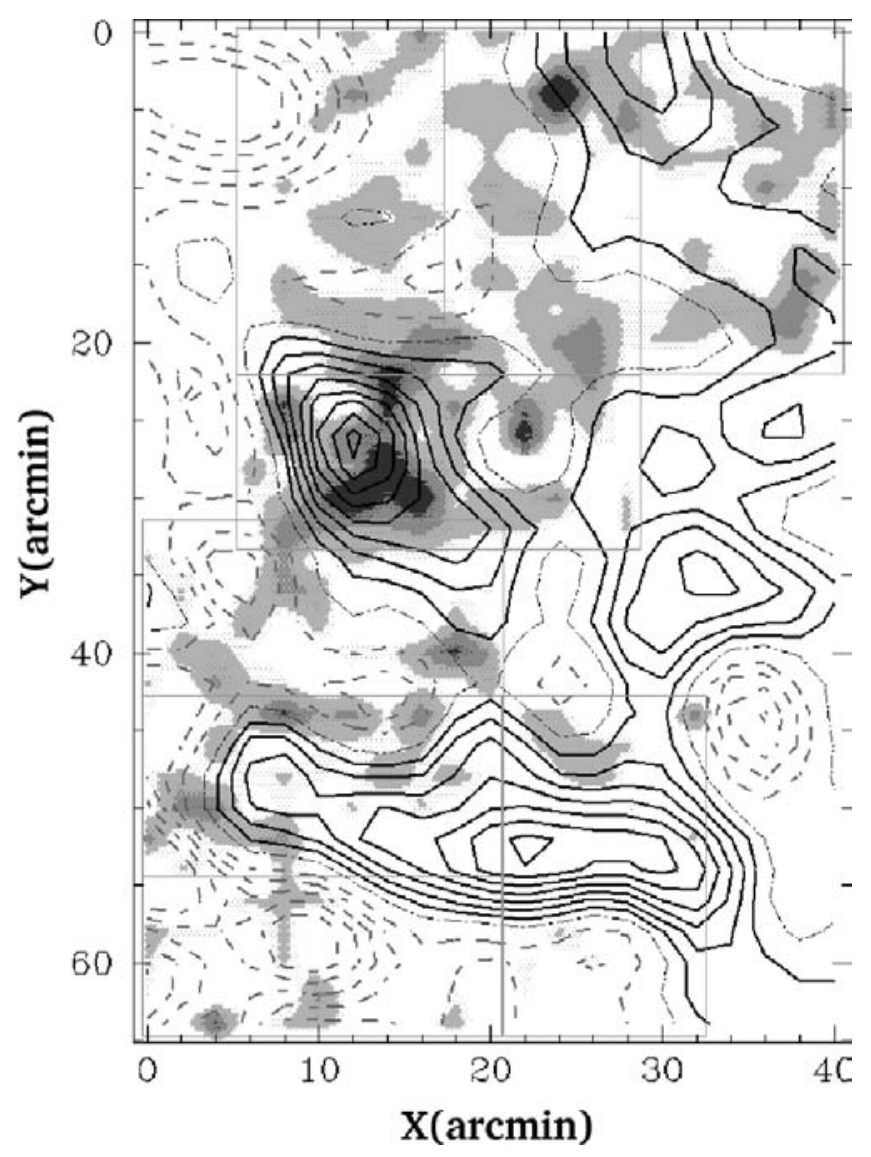

(b)

Fig. 6. The projected density of galaxies (grey scale) over the central region of the R2 ROSAT field (panel a)). The grey scale delineates regions from just above the mean level of galaxy projected density ( 4.7 galaxy $\operatorname{arcmin}^{-2}$; lightest grey) to $>7.5$ galaxy $\operatorname{arcmin}^{-2}$ (darkest grey). The diffuse soft X-ray emission from Fig. $2 \mathrm{~d}$ is overlaid as contours. The thick solid contours, thin dot-dashed contours and thick dashed contours delineate regions with extended X-ray emission above, at, and below the mean X-ray emission $\left(6 \times 10^{-3}\right.$ counts s$\left.^{-1} \operatorname{arcmin}^{-2}\right)$ respectively. The thin solid grey lines show the pattern of the wide field pointings. In panel b) the projected galaxy density is restricted to objects with measured photometric redshift $\left(\tilde{\chi}^{2}<2\right)$ and $0.3<z_{\mathrm{ph}}<0.6$. The mean galaxy projected density are at 1.4 galaxy $\operatorname{arcmin}^{-2}$ (lightest grey) while the high density regions (darkest grey) are above 2.8 galaxy $\operatorname{arcmin}^{-2}$.

and stellarity class ${ }^{6}>0.95$ ) in color-color diagrams to obtain the best agreement with the theoretical stellar main sequence. As reference we used the digital stellar spectra compiled by Pickles (1998). The required corrections were below $5 \%$.

With the zero points refined in this way we re-ran SExtractor on the images in all bands, for the objects detected in the $r^{\prime}$ band, to derive the colors of all galaxies.

The $5 \sigma$ limiting magnitude for point sources is about 23.6 in $r^{\prime}$. Even though counts are consistent with those of SDSS (Yasuda et al. 2001) and with Metcalfe et al. (2001) up to $r^{\prime}=22.5$, analysis of the differential counts within the two fields shows that the completeness in field $\mathrm{A}$ is different to that in field B. Field A is up to $80 \%$ complete at $r^{\prime}=23.3$ while field B reaches this completeness only at $r^{\prime}=22.9$. The number of objects are comparable only at magnitude 22.8 . This difference most probably arises from different seeing conditions prevailing during the observations.

\footnotetext{
${ }^{6}$ See Bertin \& Arnouts (1996).
}

We have quantified any possible galaxy overdensity associated with the diffuse X-ray filaments by using two complementary methods: 1) mapping the projected density of objects and 2) searching for clumps of galaxies.

A map of the projected density of objects was constructed by grouping all the objects in sub-sections of $2^{\prime} \times 2^{\prime}$. To avoid stellar contamination and to take into account the completeness of the galaxy catalog we have considered only those objects in magnitude range $r^{\prime}=19-22.8$.

The resulting galaxy density map is shown in grayscale in Fig. 6a, where the contours give the distribution of the diffuse soft X-ray emission (from Fig. 2d). Interestingly, the maximum galaxy density, about 10 galaxy/ $\operatorname{arcmin}^{2}$ (to be compared with the average of $\sim 4.1$ galaxy $/ \mathrm{arcmin}^{2}$ ), coincides with the maximum of the soft X-ray emission. The probability of random coincidence of the two maxima is $<1 \%$ (inferred through simulation). The second brightest filament (structure 2 in Fig. 2d) does not show an obvious associated overdensity of galaxies.

As an alternative method we have used the Voronoi tessellation (Ramella et al. 2001) to detect overdensities of galaxies 


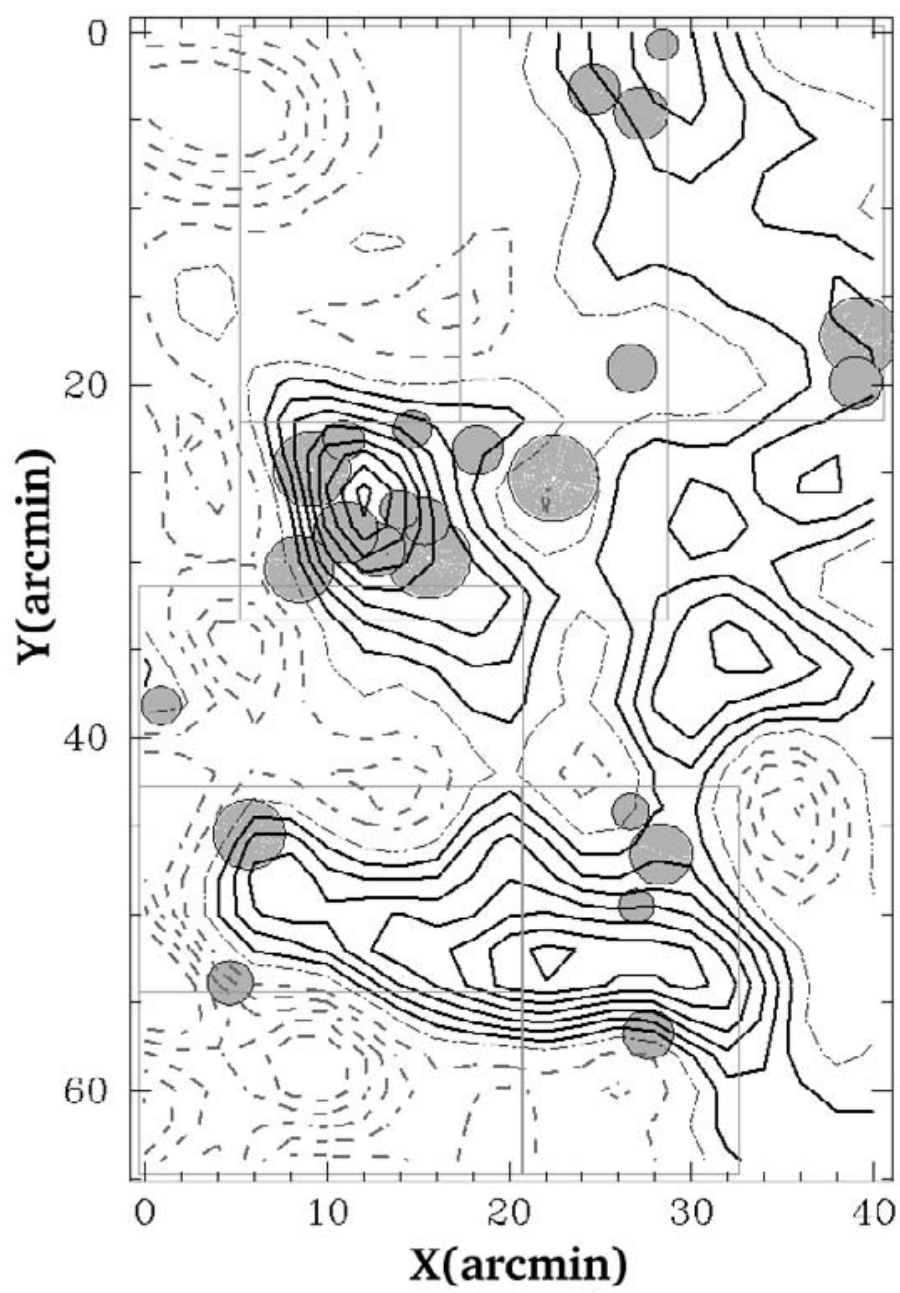

Fig. 7. Galaxy clumps found by Voronoi algorithm versus extended soft X-ray emission (contours are the same of Fig. 6). Each association of galaxies is represented by a circle (see Ramella et al. 2001). Note the correlation between the galaxy associations and the structure "1".

or clumps. By using a significance for the detection threshold of $75 \%$ and choosing a confidence level for background fluctuations of $90 \%$, we found 24 clumps in the whole field (i.e. $\mathrm{A}+\mathrm{B})$. The distribution and extension of these groups is shown in Fig. 7. There is a clear excess of clumps in the region of maximum X-ray emission (structure 1) that confirms the result of the projected density map. Some galaxy clumps are also detected around the southern filament (structure 2) and around the NW (fainter) diffuse emission, but the significance is obviously much lower.

Finally, we should mention that we have checked that the galaxies overdensities obtained in our optical maps are not due to instrumental effects or to statistical fluctuations. The former issue was tested by deriving a map of projected density of stars, and establishing that they showed no clustering or correlation with the X-ray emission, nor with the Galactic HI maps, implying that the association with the soft X-ray emission is an intrinsic characteristic of the extragalactic objects. Secondly, we run 1000 simulations of random distributions of galaxies with the goal of identifying the degree of clustering due to statistical fluctuations. Within the artificial data we found that "pixels" $\left(2^{\prime} \times 2^{\prime}\right)$ with galaxy densities in excess of 7 galaxy $\operatorname{arcmin}^{-2}$ (the dark regions in Fig. 6a) are less than $0.44 \%$ of the total distribution (upper limit at $1 \sigma$ ), while in the observation the fraction of "pixels" with densities in excess of 7 galaxy $\operatorname{arcmin}^{-2}$ is $8.4 \%$ of the total. Moreover, the "high density pixels" from the simulations are distributed randomly and not clustered as observed in our fields. The results of these simulations clearly indicate that the overdensities observed by us are not due to statistical fluctuations.

\section{The 3-D distribution of galaxies in the field}

In this section we focus on the overdensity of galaxies corresponding to structure " 1 ". We have derived galaxy photometric redshifts with the final goal of determining whether the overdensity of galaxies observed in the projected distribution is also observed in redshift space. The photometric redshifts were obtained by means of the Hyperz code (Bolzonella et al. 2000) and by exploiting the photometry from all five available filters. Within the code we used all available templates (namely ellipticals, spirals and irregulars), and we allowed for a moderate degree of extinction, up to $A_{\mathrm{v}}=0.6$. We checked that the redshift distribution is not very sensitive to these parameters. As previously stated, we limit our considerations to galaxies with $r^{\prime}$ magnitudes between 19 and 22.8, which contribute up to $54 \%$ of the entire catalog. Of these selected objects $59 \%$ have redshift fitted with $\tilde{\chi}^{2}<1$, while $73 \%$ are fitted with a $\tilde{\chi}^{2}<2$; in our analisys we have rejected objects with a photometric redshift fitted with a $\tilde{\chi}^{2}>2$. In some cases the photometric redshift implies an absolute magnitude brighter than $M_{r^{\prime}}=-23$; these are mostly objects whose photometric redshifts are fitted with $\tilde{\chi}^{2}>2$ and the high luminosity is most likely a consequence of an incorrect redshift determination. We have therefore discarded all objects with $M_{r^{\prime}}<-23$. By imposing these constraints we are left with $63 \%$ of the objects of the sample for the analysis of the photometric redshifts.

In the first two panels of Fig. 8 we show the distribution of the photometric redshifts for galaxies within the isophote at $30 \%$ from the X-ray maximum in structure " 1 " (Fig. 2), and the average redshift distribution in the field. The ratio between these two distributions is shown in the third panel. A clear excess of objects in the redshift range $0.3<z<0.6$ is seen. The excess has a high significance as shown in the fourth panel, where the excess of galaxies in each redshift bin is reported in terms of $\sigma$ (the latter inferred assuming Poisson statistics). Given the expected errors in the photometric redshifts $(\sim \pm 0.15)$ the observed overdensity is consistent with a real structure at $z \sim 0.45$. By combining the three redshift bins where the overdensity is observed, the significance of the redshift overdensity is $6 \sigma$.

In Fig. $6 b$ we show the map of galaxy density, similar to Fig. 6a, restricted to objects with measured photometric redshift $\left(\tilde{\chi}^{2}<2\right)$ and in the redshift range $0.3<z_{\text {ph }}<0.6$. The contrast between density of galaxies on the X-ray maximum relative to the field is clearly higher than in the total projected density map of Fig. 6 a.

The association of the diffuse soft X-ray structure " 1 " with a 3-D overdensity of galaxies, as inferred in the last two sections, strongly imply that the former is associated to an 


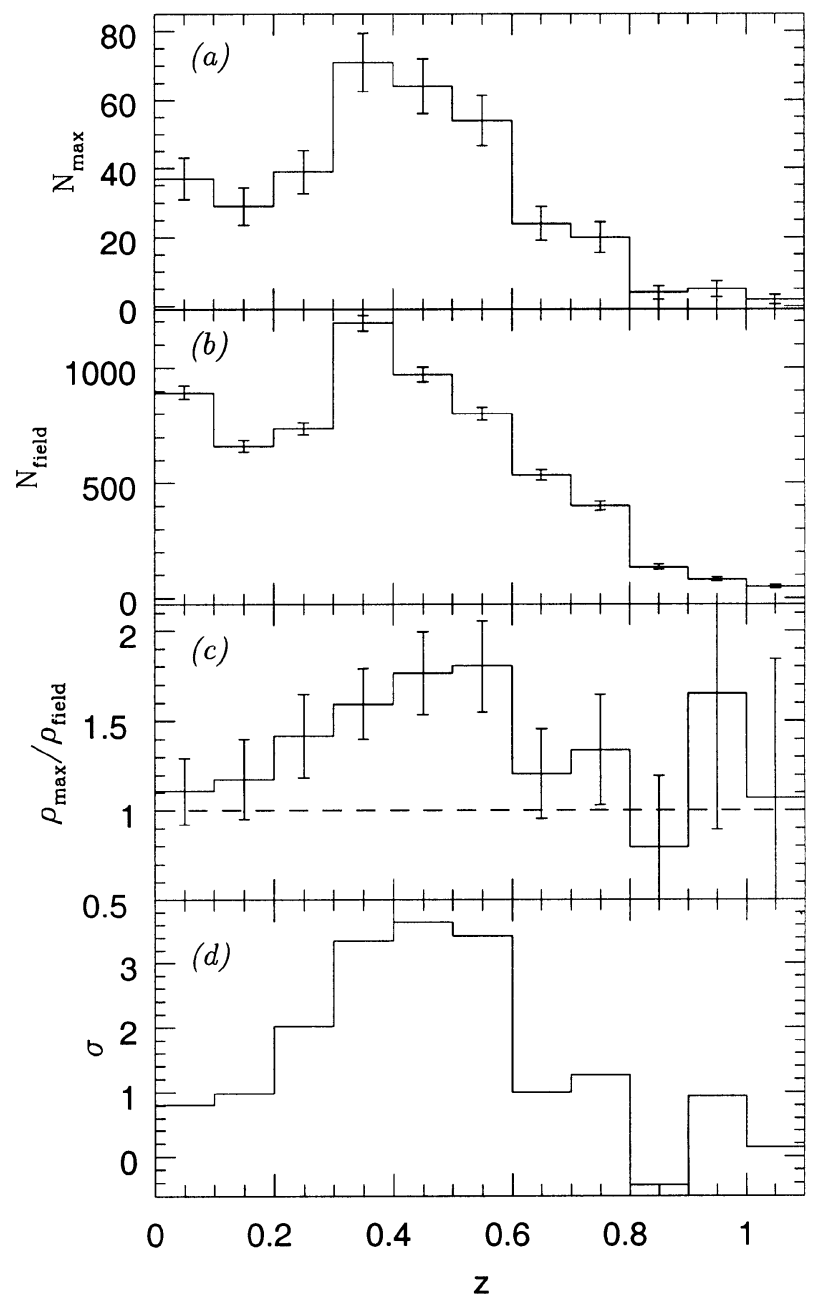

Fig. 8. Panel a) Redshift distribution of the galaxies along the $30 \%$ FWHM isophote in the region of maximum X-ray emission; b) The whole field distribution; c) Ratio between the surface densities of the two regions. In case of no overdensity the histogram should follow the dashed line, instead a clear excess of objects in the redshift range $0.3<z<0.6$. d) Excess of objects in units of $\sigma$ of the distribution.

extragalactic medium and, more specifically, at a redshift of about 0.45 .

\section{Summary and final remarks}

We have analyzed a deep ROSAT field in a region of high Galactic latitude and low Galactic HI absorption. After removal of the point sources and after correction for absorption, the softest map show evidence for diffuse/extended structures. These diffuse structures have an X-ray energy distribution that is much softer than expected from clusters, groups or unresolved emission from AGNs, but it is consistent with emission from the diffuse warm intergalactic medium expected by the cosmological models, both in terms of shape (plasma at $k T \sim$ $0.3 \mathrm{keV})$ and of flux $\left(\left\langle F_{0.2-0.3 \mathrm{keV}}\right\rangle \approx 10^{-12} \mathrm{erg} \mathrm{cm}^{-2} \mathrm{~s}^{-1} \mathrm{deg}^{-2}\right)$.

To discriminate between a Galactic or extragalactic nature of the diffuse gas we have correlated the soft X-map with multiband optical images in this field. The most prominent diffuse X-ray structure in the ROSAT map appears associated with an overdensity of galaxies at a photometric redshift of $\sim 0.45$. This association, along with the X-ray properties of the former (Sect. 2), strongly suggest that we are observing an extragalactic structure most likely tracing the warm intergalactic medium predicted by cosmological theories, in this case at redshift $\sim 0.45$, which is expected to be the main reservoir of baryonic matter at low redshifts.

The second most prominent diffuse X-ray structure " 2 " is not associated, in our optical maps, with a pronounced galaxy overdensity, although there are some clumps of galaxies surrounding it (Fig. 7). Either this structure is not extragalactic (e.g. associated to our Galactic halo) or it is associated with galaxies at redshift higher than 0.8 , where most galaxies escape detection in our optical images. Finally, this may be a case of warm baryonic gas at relatively low redshift, not enclosing galaxy overdensities, but possibly bridging those few galaxy clumps detected in its surrounding.

Multi-object spectroscopy should provide a critical test on the nature of these diffuse structures. Indeed the spectroscopic information would both confirm the galaxy redshift distribution and give an estimate of the involved virial masses to be compared with the temperature inferred for the barionic gas.

Acknowledgements. We are grateful to R. Croft for providing us with the unpublished maps of his simulations and to M. Bolzonella for making available her Hyperz code and for providing additional information and suggestions. We thank G. Hasinger for helpful comments during the early stages of this work. We are also grateful to the staff of Isaac Newton Telescope and of the Effelsberg 100 meter telescope for their support during the observations. We are grateful to Peter Kalberla for giving us access to Effelsberg calibration software and Alison Peck for help with the observations.

\section{References}

Bagchi, J., Ensslin, T. A., Miniati, F., et al. 2002, New Astr., 7, 249 Bertin, E., \& Arnouts, S. 1996, A\&AS, 117, 393

Bolzonella, M., Miralles, J.-M., \& Pelló, R. 2000, A\&A, 363, 476

Bond, J. R., Kofman, L., \& Pogosyan, D. 1996, Nature, 380, 603

Brinkmann, W., Laurent-Muehleisen, S. A., Voges, W., et al. 2000, A\&A, 356, 445

Bryan, G. L., \& Voit, G. M. 2001, ApJ, 556, 590

Burles, S., \& Tytler, D. 1998, ApJ, 499, 699

Cen, R., \& Ostriker, J. P. 1999, ApJ, 514, 1

Davé, R., Cen, R., Ostriker, J. P., et al. 2001, ApJ, 552, 473

Fukugita, M., Hogan, C. J., \& Peebles, P. J. E. 1998, ApJ, 503, 518

Kalberla, P. M. W., Mebold, U., \& Reich, W. 1980, A\&A, 82, 275

Kalberla, P. M. W., Mebold, U., \& Reif, K. 1982, A\&A, 106, 190

Kull, A., \& Böhringer, H. 1999, A\&A, 341, 23

Kuntz, K. D., Snowden, S. L., \& Mushotzky, R. F. 2001, ApJ, 548, L119

Landolt, A. U. 1992, AJ, 104, 340

Lockman, F. J., Jahoda, K., \& McCammon, D. 1986, ApJ, 302, 432

Metcalfe, N., Shanks, T., Campos, A., McCracken, H. J., \& Fong, R. 2001, MNRAS, 323, 795

Mittaz, J. P. D., Carrera, F. J., Romero-Colmenero, E., et al. 1999, MNRAS, 308, 233

Pettini, M., \& Bowen, D. V. 2001, ApJ, 560, 41 
Phillips, L. A., Ostriker, J. P., \& Cen, R. 2001, ApJ, 554, L9

Pickles, A. J. 1998, VizieR Online Data Catalog, 611, 00863

Ramella, M., Boschin, W., Fadda, D., \& Nonino, M. 2001, A\&A, 368, 776

Rauch, M., Miralda-Escude, J., Sargent, W. L. W., et al. 1997, ApJ, 489, 7

Scharf, C., Donahue, M., Voit, G. M., Rosati, P., \& Postman, M. 2000, ApJ, 528, L73

Snowden, S. L., Egger, R., Finkbeiner, D. P., Freyberg, M. J., \& Plucinsky, P. P. 1998, ApJ, 493, 715

Snowden, S. L., McCammon, D., Burrows, D. N., \& Mendenhall, J. A. 1994, ApJ, 424, 714
Soltan, A. M., Hasinger, G., Egger, R., Snowden, S., \& Truemper, J. 1996, A\&A, 305, 17

Tittley, E. R., \& Henriksen, M. 2001, ApJ, 563, 673

Vikhlinin, A., McNamara, B. R., Forman, W., et al. 1998, ApJ, 502, 558

Walter, R., \& Fink, H. H. 1993, A\&A, 274, 105+

Wang, Q. D., \& McCray, R. 1993, ApJ, 409, L37

Warwick, R., Hutchinson, I., Willingale, R., Kuntz, K., \& Snowden, S. L. 1998, in Lect. Notes Phys., 506, 321

Yasuda, N., Fukugita, M., Narayanan, V. K., et al. 2001, ApJ, 122, 1104 